\title{
Refinement of a Concept Inventory to Assess Conceptual Understanding in Civil Engineering Fluid Mechanics
}

\section{Dr. Mary Katherine Watson, The Citadel, The Military College of South Carolina}

Dr. Mary Katherine Watson is currently an Assistant Professor of Civil and Environmental Engineering at The Citadel. Prior to joining the faculty at The Citadel, Dr. Watson earned her PhD in Civil and Environmental Engineering from The Georgia Institute of Technology. She also has BS and MS degrees in Biosystems Engineering from Clemson University. Dr. Watson's research interests are in the areas of engineering education and biological waste treatment.

\section{Amber Renee Mills, The Citadel}

Amber Mills is a junior Civil Engineering major at The Citadel. Originally from North Carolina, she is the student chapter president of the Society of Women Engineers and participates as a varsity athlete. She plans to pursue a graduate degree in Civil Engineering.

\section{Dr. Kevin C Bower P.E., The Citadel}

Dr. Kevin Bower is an Associate Professor and Head of the Department of Civil and Environmental Engineering at The Citadel, Charleston, South Carolina. Dr. Bower's teaching research interests are in improving active learning environments and the development of classroom pedagogy to improve moral development in engineering students.

\section{Dr. Kenneth Brannan, The Citadel}

Ken Brannan is a Professor in the Department of Civil and Environmental Engineering at The Citadel. He has served as Chair of the First-Year Programs Division and the Environmental Engineering Division and as President of the Southeastern Section of ASEE. He earned B.C.E and M.S. degrees from Auburn University and the Ph.D. from Virginia Tech. His interests include wastewater treatment and teaching and learning in engineering education.

\section{Dr. Michael H. Woo, The Citadel}

Associate Professor in Civil Engineering.

\section{Dr. Ronald W. Welch P.E., The Citadel}

Ron Welch (P.E.) received his B.S. degree in Engineering Mechanics from the United States Military Academy in 1982. He received his M.S. and Ph.D. degrees in Civil Engineering from the University of Illinois, Champaign-Urbana in 1990 and 1999, respectively. He became the Dean of Engineering at The Citadel on 1 July 2011. Prior to his current position, he was the Department Head of Civil Engineering at The University of Texas at Tyler from Jan 2007 to June 2011 as well as served in the Corps of Engineers for over 24 years including eleven years on the faculty at the United States Military Academy. 


\title{
Revising a Concept Inventory to Assess Conceptual Understanding in Civil Engineering Fluid Mechanics
}

\begin{abstract}
Given the need for civil engineers to develop solutions to society's infrastructure challenges, they must possess a deep understanding of engineering fundamentals so that they can tailor design solutions to meet growingly-complex constraints. Since research suggests that student conceptual development is enhanced through employment of engaging classroom practices, a variety of active pedagogies are being implemented in engineering education. Consequently, there is a need for assessment tools to monitor the learning outcomes of these educational interventions. Concept inventories have emerged as quick tools for examining conceptual understanding in a variety of engineering domains. While a concept inventory has been developed for fluid mechanics, it was designed for application in mechanical engineering classes. Thus, the goal of this project was to complete preliminary steps for creation of a civil engineering fluid mechanics concept inventory.

Using an expert panel and student input, concept inventory items were systematically evaluated for applicability in civil engineering courses. The expert panel reviewed items using a modified Delphi-process, while student input and performance on the existing concept inventory were used to validate outcomes from the expert panel. Results generally concurred that topics related to fluid statics, pressure measurement, conservation of mass, Bernoulli's equation, and conservation of momentum were important for civil engineers. In contrast, compressible flow and boundary effects were generally classified as beyond the scope of a civil engineering course. In addition, participants suggested that additional questions related to viscous flow in pipes and open channel flow should be added to a future civil engineering fluid mechanics concept inventory.
\end{abstract}

\section{Introduction}

Civil engineers are called upon to develop solutions to society's infrastructure-related challenges. This service is becoming increasingly more difficult, due to competing design constraints, such as minimizing cost, reducing non-renewable resource consumption, and optimizing end-user satisfaction ${ }^{1}$. As a result, civil engineers may need to deviate from well-established design templates and heuristics to develop truly innovative designs. To accomplish this feat, engineers must possess a deep conceptual understanding of engineering fundamentals so that they are able to critically analyze new scenarios and create tailored solutions ${ }^{2}$. Indeed, Streveler and colleagues ${ }^{3}$, summarizing the work of Sheppard et al. ${ }^{4}$, state that the practice of engineering involves three components: (1) problem solving, which includes processes used to define and solve problems, (2) specialized knowledge that facilitates problem solving, and (3) integration of 
problem-solving processes and knowledge. Consequently, conceptual knowledge is a prerequisite for engaging in authentic design.

As a result, many engineering educators are striving to apply and assess innovative pedagogies to encourage deep learning among their students, rather than rote memorization of facts and solution procedures ${ }^{5}$. Research has shown that despite passing grades, many engineering students lack a true understanding of subject material in a variety of courses ${ }^{2}$. Given that literature in educational psychology suggests that students construct knowledge as a result of experiences and interactions with others ${ }^{6-9}$, engineering educators are turning to more engaging teaching and learning practices to improve students' knowledge networks, as well as identify and correct misconceptions $^{10}$. Assessments for evaluating the efficacy of student-centered educational interventions, such as problem-based learning, flipped-classrooms, and collaborative learning, are greatly needed.

Concept inventories, which are highly-sophisticated, multiple-choice assessments used to capture students' conceptual knowledge in a specific engineering or science domain, can be used to identify common student misconceptions, as well as to characterize the efficacy of new teaching strategies ${ }^{11}$. Concept inventories have been developed for a variety of engineering courses, although the fluid mechanics concept inventory (FMCI) is among those of specific interest to civil engineering (CE) faculty ${ }^{12}$. However, the existing FMCI caters to mechanical engineering (ME) courses, since it includes concepts such as compressible flow ${ }^{13}$. Thus, there is a need to refine the ME-FMCI for application in CE courses.

A study was conducted to modify the ME-FMCI for use in CE courses. Each item was reviewed by a panel of fluid mechanics content experts using a modified Delphi process, which is a structured communication process that facilitates consensus building among experts ${ }^{14-16}$, as well as a fluid mechanics student. In addition, the ME-FMCI was administered to two sections of junior-level CE students before and after completion of a fluid mechanics course. Using this data, the objectives were to:

1. Collect and analyze experts' opinions on the applicability of existing ME-FMCI questions for civil engineering applications.

2. Use student performance and opinions to validate the retention or omission of existing MEFMCI questions for a future civil engineering FMCI (CE-FMCI).

\section{Background Information}

\section{Conceptual Knowledge}

Conceptual knowledge goes beyond just knowing facts and concepts. While conceptual knowledge certainly includes "knowledge about facts, [generalizations], and principles" 17" (pg. 
123)," it also encompasses how those elements' are organized ${ }^{3,17,18}$. Rittle-Johnson ${ }^{18}$ states that conceptual knowledge includes "understanding of principles governing a domain and the interrelations between units of knowledge in a domain" (pg. 2). Similarly, Starr ${ }^{19}$ describes that conceptual understanding must be "deep" and "rich with connections" (pg. 408). In fact, Bransford et $\mathrm{al}^{20}$. describe that experts structure knowledge around central ideas, while Alexander and Murphy ${ }^{21}$ support that expertise requires development of knowledge "coherently organized around key domain concepts and principles" (pg. 566). Consequently, conceptual knowledge is factual, yet structured, complex, and interrelated.

Acquiring conceptual knowledge has been described as more than just memorizing facts, but indicates that the learner knows something. For instance, Montfort et al. ${ }^{2}$ describes that conceptual knowledge is "internal" and relates knowledge with intuition, stating that "you don't remember something you understand conceptually, it is just true" (pg. 111). Similarly, Streveler et al. ${ }^{3}$ recounts that conceptual knowledge provides engineers with "engineering judgment" or "heuristic thinking" that is necessary for them to make real-time decisions in the absence of advanced computations. Clearly, conceptual knowledge is a sign of more significant learning and cannot be obtained through rote memorization.

Completion of procedural computations does not demonstrate conceptual knowledge. For instance Montfort et al. ${ }^{2}$ presents that conceptual understanding requires that students grasp the underlying significance of their computations, rather than simple demonstration of their abilities to complete steps. Educational practices common in engineering, such as problem-based lectures, homework, and exams, have been shown to improve students' abilities to complete calculations, while true conceptual understanding is often more difficult to achieve ${ }^{2,22}$. Conceptual understanding is more valuable than the ability to execute a sequence of steps because it tends to be transferable to new situations and contexts ${ }^{2}$. Bruner, the famous cognitive psychologist, summarizes this argument by stating that mastery is "the development of an attitude toward learning and inquiry, toward guessing and hunches, toward the possibility of solving problems on one's own" (pg. 20).

While the ability to perform calculations does not prove conceptual understanding, conceptual knowledge can enable effective problem solving. Summarizing the work of Rittle-Johnson et al. $^{23}$, Streveler et al. ${ }^{3}$ concludes that conceptual knowledge can enable students to identify mistakes in their procedures, as well as create new approaches through correctly identifying and sequencing aspects from known procedures. Thus, conceptual knowledge is important for engineering "competence" (pg. 279), since engineers are inherently problem solvers that must constantly analyze new contexts ${ }^{4}$. 


\section{Misconceptions and Conceptual Change}

Given the role of conceptual knowledge in engineering problem solving, it is important to ensure that students develop accurate conceptual frameworks. Piaget ${ }^{24}$ proposed that knowledge is organized into different categories (or schema), and that when students encounter new concepts during learning, they must either assign them to existing categories or reconfigure categories to accommodate the new concepts. One viewpoint is that reclassifying a concept among categories is what defines conceptual change ${ }^{2,25}$. Misconceptions, which are deeply-held, prior conflicting beliefs, can make conceptual change particularly difficult ${ }^{10}$. Chi and collaborators hold that all "entities" belong to different "ontological categories," which include matter, processes, and mental states ${ }^{26}$ (pg. 28). For instance, many scientific concepts belong to the processes category, but confusion arises because these processes involve matter. Chi et al. ${ }^{26}$ use the concept of electrical current, which is created when a charged particle moves in an electric field, to demonstrate misunderstanding among categories. Students may believe that because electric current includes matter (i.e. charged particles, wires, etc.), it itself is also matter. However, electric current is a process that involves different types of matter. The process of categorical shift is so difficult, that it can seldom be accomplished through lecturing alone ${ }^{22}$.

A conceptual framework plagued with misconceptions can prevent students from integrating new knowledge during the learning process ${ }^{20}$; thus, educators must tailor their teaching and learning strategies to address students' prior knowledge. If students simply have no or incomplete prior knowledge about a topic, then enrichment strategies are needed to help students add knowledge

or fill the gaps, respectively ${ }^{10,27}$. However, if students have incorrect prior knowledge related to the subject matter, then students must be guided in the process of discovering and correcting their misconceptions ${ }^{28}$. Some misconceptions can be corrected by refuting false beliefs or transforming mental models, while the categorical shifts discussed above can be significantly more difficult to achieve ${ }^{10}$. For this most extreme case ${ }^{26}$, careful steps must be taken to ensure

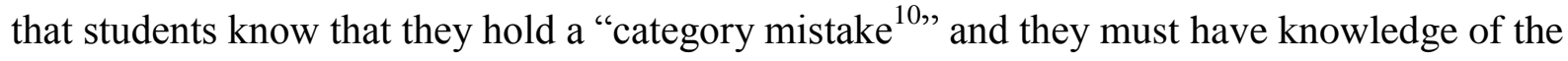
correct category (pg. 77). Overall, students' prior knowledge must be assessed, since misconceptions can prevent the development conceptual understanding.

\section{Concept Inventories}

Given the importance of conceptual knowledge and the potential for misconceptions, there is a clear need to be able to assess conceptual understanding and monitor conceptual change. Originating in the physics education literature ${ }^{29}$, concept inventories have become increasingly popular as rapid ${ }^{3}$ tools in engineering education for capturing conceptual understanding. Concept inventories are carefully-designed, multiple-choice instruments that require students to select the correct answer for a particular problem among distractors based on known student misconceptions ${ }^{30}$. In addition to the famous force concept inventory $(\mathrm{FCI})^{29}$, members of the 
Foundation Coalition have introduced a variety of assessments for domains within engineering, including circuits, computer engineering, electromagnetics, electronics, signals and systems, waves, dynamics, fluid mechanics, heat transfer, strength of materials, and thermodynamics ${ }^{31}$.

Design of a concept inventory is usually based on expert consensus concerning common misconceptions held by students in a particular subject ${ }^{30}$. One approach for accomplishing this task is the Delphi method, which is a structured communication process that facilitates consensus building among experts about a complex topic or problem ${ }^{14-16}$. While there are many types of Delphi procedures reported in the literature, most use multiple iterations of questionnaires to collect opinions from participants. Throughout these iterations, controlled feedback, in which respondents' scores and comments are compiled and distributed to other participants, is practiced $^{14}$. Furthermore, judges are afforded some degree of anonymity during the process ${ }^{14}$. This controlled communication process is important for encouraging experts to engage in independent thought and analysis ${ }^{15}$. While the original Delphi process was developed the Rand Corporation ${ }^{16}$ in the 1950s, it has since been used in a number of engineering education studies ${ }^{32,}$ ${ }^{33}$, including the creation of concept inventories ${ }^{11,13,33,34}$.

Development and use of concept inventories has revealed important insights about the state of engineering and science education. Foremost, students may lack a real conceptual understanding of engineering course matter, despite acceptable grades and passing rates ${ }^{2}$. Instructor-centered pedagogies enacted in many technical classrooms may lead to low conceptual gains, as measured by concept inventories ${ }^{35}$. In fact, students participating in traditional lecture-based courses typically experience learning gains of only approximately $30 \%{ }^{30}$, while those engaged in more interactive courses achieve learning gains of closer to $50 \%$, on average ${ }^{35}$. Others agree that disregard for students' needs to construct their own knowledge, as well as the detrimental effect of incorrect prior knowledge on learning, likely contribute to poor quality of conceptual knowledge ${ }^{2}$. Given the potential for improvement in technical education, many researchers and educators are using concept inventories as tools for identifying misconceptions and tracking the efficacy of educational materials and interventions ${ }^{36,37}$.

\section{Fluid Mechanics Concept Inventory}

A fluid mechanics concept inventory (FMCI) was developed by three mechanical engineering faculty (T. Newell, University of Illinois, J. K. Martin and J. W. Mitchell, University of Wisconsin) in 2003, based on knowledge deemed essential by faculty and students (Table 1). Developers of the FMCI cite that compilation of topics was likely more difficult than for the original force concept inventory, since fluid mechanics is "a combination of a large number of somewhat disparate concepts ${ }^{12}$ " (pg. 23). Faculty developed a list of more than 25 different concepts, which comprised three different categories: basic concepts (i.e. properties), fundamental fluid relations (i.e. conservation of mass and momentum), and special cases (i.e. 
pipe networks and turbomachinery). Once the set of key topics was shortened, in part using student input, a set of questions was developed. The items were created to be completed without a calculator and with included visual representations, as was done for the original force concept inventory $^{12,13}$.

The FMCI underwent several rounds of revisions and was also used for assessment of mechanical engineering courses at the University of Wisconsin at Madison. Among four sections of fluid mechanics course, average learning gains ranged from 18-30\% for all items (equation 1). Learning gains varied significantly based on course section, but the greatest gains were seen for problems related to basic concepts, conservation of mass, and ideal conservation of momentum. In contrast, lowest gains were observed for viscous conservation of momentum, hydrodynamics, and conservation of energy. Additional analysis showed only slight correlation between course grades and performance on the inventory. Some faculty reported concerns that students did not perform at their best, since each student only received a homework grade for completed answer sheet. Overall inventory results were used to improve course instruction to enhance student conceptual development ${ }^{38}$.

Where,

$$
\text { Learning Gain }=\left[(\text { Post }- \text { Pre }) /(\text { No. Questions }- \text { Pre })^{*} 100\right]
$$

Post $=$ Number of items answered correctly on the post-test.

Pre $=$ Number of items answered correctly on the pre-test

No. Questions = Total number of questions on the concept inventory.

Table 1. Concepts tested on existing FMCI $^{1}$.

\begin{tabular}{clcl}
\hline No. & Targeted Concepts & No. & Targeted Concepts \\
\hline 3 & Continuity; compressible & 18 & Manometry; compressible \\
4 & Bernoulli; incompressible & 19 & Drag force; compressible \\
5 & Boundary conditions & 20 & Boundary layer; compressible \\
6 & Momentum; incompressible & 21 & Boundary layer; incompressible \\
7 & Pressure definition & 22 & Continuity; incompressible \\
8 & Boundary layers; incompressible & 23 & Continuity/Bernoulli; incompressible \\
9 & Pascal's Law & 24 & Boundary layer; compressible \\
10 & Manometry; compressible & 25 & Impulse-momentum; incompressible \\
11 & Bernoulli; incompressible & 26 & Boundary layer; compressible \\
12 & Forces on submerged surface & 27 & Continuity/Bernoulli; incompressible \\
13 & Ideal Gas Law & 28 & Drag force; compressible \\
14 & Manometry; compressible & 29 & Drag force; compressible \\
15 & Shear stress; compressible & 30 & Pressure measurement; compressible \\
16 & Boundary layers & 31 & Continuity/Temperature variations; compressible \\
17 & Bernoulli; incompressible & 32 & Fluid properties (viscosity) \\
\hline
\end{tabular}

${ }^{\mathrm{I}}$ The final FMCI is available from John Mitchell (mitchell@engr.wisc.edu). 


\section{Study Methods}

\section{Gathering Data from Expert Panel}

A modified, two-phase Delphi process was used to gather expert opinions on the appropriateness of FMCI items to civil engineering courses. In the first round, four CE fluid mechanics content experts were asked to review each of the existing FMCI items. Judges used binary responses (yes/no) to indicate whether or not each question should be included on a CE concept inventory. In addition, judges documented their rationale for each designation, including each item's relevance to a set of course objectives from an actual CE fluid mechanics course (Table 2). Responses were sent to the lead judge who anonymously compiled all responses. This process was repeated twice.

Table 2. Course objectives for a civil engineering fluid mechanics course.

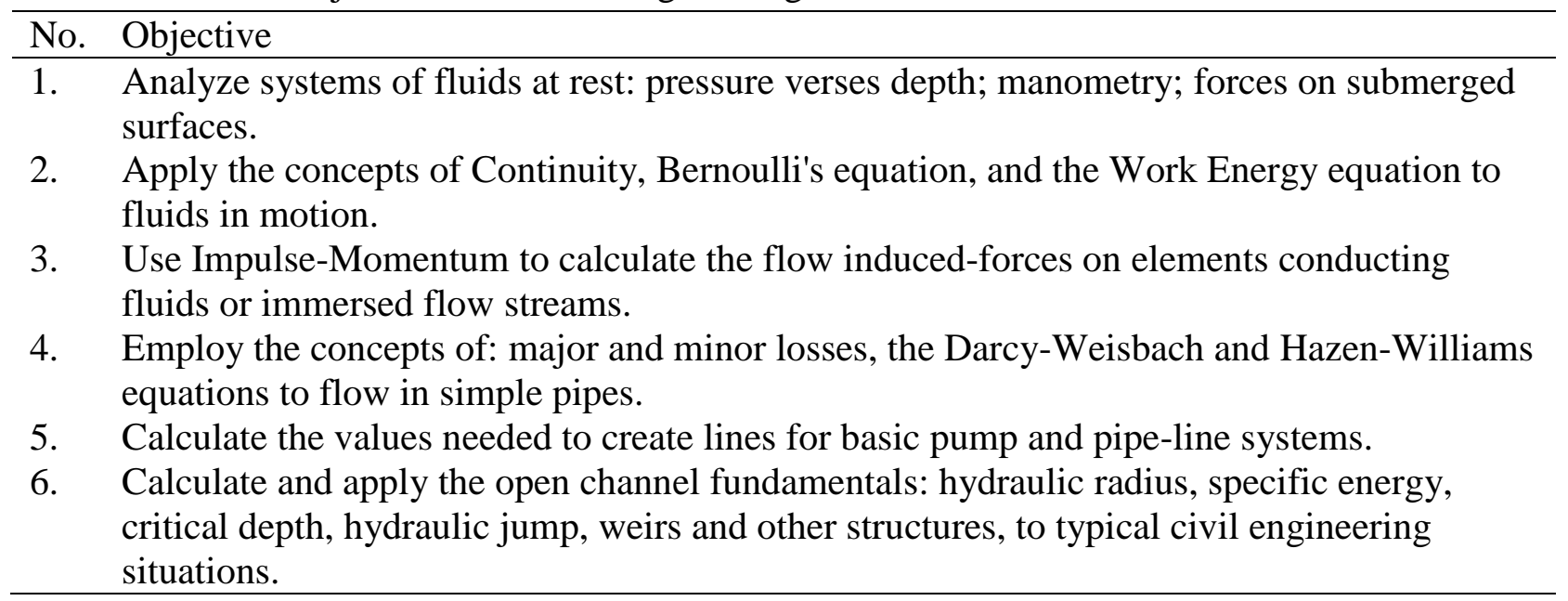

\section{Analyzing Student Performance on the Fluid Mechanics Concept Inventory}

Student performance on the existing FMCI was used to validate outcomes from the expert panel. Junior civil engineering students $(n=35)$ enrolled in a fluid mechanics course at a teachingfocused college in the southeastern United States (US) completed the FMCI at the beginning and end of the Fall 2013 semester. The course was designed to achieve a set of learning objectives (Table 2) through the use of short lectures, instructor- and peer-assisted problem solving sessions, homework sets, and exams. Student responses to each FMCI item were coded as "correct" or "incorrect" and McNemar's test ${ }^{39}$ for paired, nominal data was used to identify any changes in student performance between pre- and post-test administrations. Significant trends were identified for $p \leq 0.05$. 


\section{Collecting a Student Perspective on the Fluid Mechanics Concept Inventory}

Since student insights are recommended in development of concept inventories ${ }^{12,40}$, a highperforming civil engineering student, who had just completed a fluid mechanics course, was asked to review the FMCI. Like the expert judges, she examined each of the existing FMCI items and used binary responses (yes/no) to indicate whether or not each question should be included on a CE concept inventory. The student also provided written support for her decision and mapped each relevant item to a set of course objectives. Her responses were used in conjunction with the expert panel and actual student performance on the FMCI to determine which items should be retained on future versions of the Inventory.

\section{Results}

\section{Outcomes from Expert Panel}

Using a two-phase communication process, experts systematically reviewed existing ME-FMCI to identify relevant items for a CE-FMCI. Participants also brainstormed on important CE concepts not appearing on the ME-FMCI. The results from each round of expert review are presented sequentially followed by an analysis of the additional topics suggested by the experts.

\section{First Round Consensus}

Upon the first round of individual evaluation of the FCMI, judges were in agreement about the relevance of most items to civil engineering courses (Krippendorff's alpha $=0.654)($ Table 3 ). For instance, judges agreed that questions related to fluid statics (e.g. Pascal's Law, manometry, forces on submerged surfaces), fluid dynamics (e.g. Bernoulli's, impulse-momentum, and continuity equations), and basic definitions (e.g. viscosity and pressure) should be retained on the assessment. On the contrary, judges concurred that questions related to boundary conditions and drag effects for compressible fluids were not essential for civil engineers.

Final Round - Compressible Flow

Consensus among judges improved after the second round of evaluation (Krippendorff's alpha $=$ 0.918). Many of the FCMI items that were re-visited during Round 2 were related to compressible flow (Table 3). Based on Round 1 comments, it was evident that compressible flow should be represented on the CE-FMCI, although boundary layer phenomena of gasses were beyond the scope of a civil engineering course (No. 20, 24, 26) (Figure 2B). However, questions that could be correctly answered using principles applicable to both compressible and incompressible flow were retained. For instance, one question required students to analyze flow of a compressible fluid with changing density in a pipe using conservation of mass (No. 3). Similarly, judges agreed that manometer problems $($ No. 14, 18) involving gasses were appropriate, because analysis involved simple knowledge of hydrostatic pressure. Finally, one 
fluid measurement problem was retained because it required knowledge of stagnation and dynamic pressures of air, two concepts which are also applicable to incompressible flow (No. 30).

Final Rounds - Boundary Layers

Although compressible boundary layer questions were omitted, similar problems involving incompressible flows were retained (Table 3). The retained problems required students to identify the velocity profile within an incompressible fluid trapped between two moving or stationary plates (No. 16, 21) (Figure 1C). Velocity profiles provided could be interpreted as shear stress versus shear strain diagrams. For Newtonian fluids, the slope of the linear shear stress versus shear strain diagram is equal to the viscosity of the fluid. Consequently, knowledge of viscosity could be applied by civil engineering students to correctly answer items 16 and 21 .

\section{Additional Civil Engineering Topics}

In addition to evaluating existing ME-FMCI items, judges also brainstormed about topics that may be included on a future CE-FMCI. All judges included some aspect of viscous flow in pipes, including major/minor losses, turbulent flow, use of machinery (pumps/turbines) and energy/hydraulic gradelines (course objectives 4 and 5; Table 2). While three judges suggested that concepts related to open channel flow should be included on a CE-FMCI, one judge proposed that this topic is really more appropriate for a hydraulics class.

\section{Analysis of Student Performance on Concept Inventory}

To validate outcomes from the expert panel, CE student performance on the ME-FMCI was analyzed with the hypothesis that students would perform well on items that judges designated as "retain" and poorly on those that were recommended for omission.

\section{Examination of Student Performance After Course Completion}

Several aspects of student performance on the FMCI aligned with judges' consensus on the applicability of items for civil engineers. Of the 12 items that were designated by judges as "omit," half were answered correctly by only $25 \%$ of students or less (No. 10, 13, 15, 19, 26, 31) (Figure 2C). All of these low-performance problems focused on some aspect of compressible flow, including topics of shear stress, boundary layers, and temperature variations (Table 4).

\section{Examination of Items Impacted by Course Completion}

To further support the validity of the judges' consensus, all of the items that significantly improved between pre and post assessments were designated to be retained in future versions of the inventory. Topics that were positively influenced by completion of the course largely focused on incompressible flow, including application of the continuity equation (No. 23, 27), Bernoulli's principle (No. 4, 17, 27) (Figure 1A), and the impulse-momentum equation (No. 6) 
(Figure 1B). In addition, conceptual understanding of pressure significantly increased $(p \leq 0.01)$ after participation in the fluid mechanics course, including the definition of pressure (No. 7) and Pascal's Law (No. 9). Given the agreement between the judges and students, it is clear that these items should be included on the revised inventory (Table 4).

Table 3. Outcomes from expert panel evaluations on relevance of ME-FMCI items for civil engineering.

\begin{tabular}{|c|c|c|c|c|}
\hline No. & Targeted Concepts & $\begin{array}{l}\text { Learning } \\
\text { Objective }\end{array}$ & Round 1 & Round 2 \\
\hline 3 & Continuity; compressible & 2 & & Retain \\
\hline 4 & Bernoulli; incompressible & 2 & Retain & \\
\hline 5 & Boundary conditions & N/A & & Omit \\
\hline 6 & Momentum; incompressible & 3 & Retain & \\
\hline 7 & Pressure definition & 1 & Retain & \\
\hline 8 & Boundary layers; incompressible & N/A & & Omit \\
\hline 9 & Pascal's Law & 1 & Retain & \\
\hline 10 & Manometry; compressible & $\mathrm{N} / \mathrm{A}$ & & Omit \\
\hline 11 & Bernoulli; incompressible & 2,4 & Retain & \\
\hline 12 & Forces on submerged surface & 1 & Retain & \\
\hline 13 & Ideal Gas Law & N/A & Omit & \\
\hline 14 & Manometry; compressible & 1 & Retain & \\
\hline 15 & Shear stress; compressible & N/A & Omit & \\
\hline 16 & Boundary layers & $0^{1}$ & & Retain \\
\hline 17 & Bernoulli; incompressible & 2 & & Retain \\
\hline 18 & Manometry; compressible & 1 & Retain & \\
\hline 19 & Drag force; compressible & $\mathrm{N} / \mathrm{A}$ & Omit & \\
\hline 20 & Boundary layer; compressible & N/A & Omit & \\
\hline 21 & Boundary layer; incompressible & $0^{1}$ & & Retain \\
\hline 22 & Continuity; incompressible & 2 & Retain & \\
\hline 23 & Continuity/Bernoulli; incompressible & 2 & Retain & \\
\hline 24 & Boundary layer; compressible & N/A & Omit & \\
\hline 25 & Impulse-momentum; incompressible & 3 & Retain & \\
\hline 26 & Boundary layer; compressible & N/A & Omit & \\
\hline 27 & Continuity/Bernoulli; incompressible & 2 & & Retain \\
\hline 28 & Drag force; compressible & $\mathrm{N} / \mathrm{A}$ & Omit & \\
\hline 29 & Drag force; compressible & $\mathrm{N} / \mathrm{A}$ & Omit & \\
\hline 30 & Pressure measurement; compressible & 1,2 & & Retain \\
\hline 31 & Continuity/Temperature variations; compressible & N/A & & Omit \\
\hline 32 & Fluid properties (viscosity) & $0^{1}$ & Retain & \\
\hline
\end{tabular}

${ }^{\mathrm{I}}$ There is no explicit learning objective related to fluid properties, although subsequent learning objectives require understanding of basic properties.

Student performance on one item significantly decreased $(p \leq 0.05)$ after completion of the fluid mechanics course. The question required students to compare the forces resulting from impaction of a structure by an incompressible fluid (No. 25) (Figure 1D). Impulse-momentum 
was extensively addressed in the fluid mechanics course, and students even improved their accuracy in answering an earlier question related to impulse-momentum (No. 6) (Figure 1B). It is predicted that student fatigue contributed to the poor performance on item No. 25, since this question was near the end of the inventory. Reducing the length of the instrument and eliminating questions that are unfamiliar to students are expected to improve performance on questions near the end of the test (Table 4).

\section{Student Commentary on Concept Inventory}

\section{Consensus with Expert Judges}

A high-performing student having just completed a CE fluid mechanics course, designed to accomplish the sample learning objectives (Table 2), also evaluated each item on the existing FMCI (Table 4). The student agreed with the judges on 24 out of 30 of the concept inventory items. Specifically, they agreed that topics related to fluid statics, fluid dynamics, and basic definitions related to incompressible fluids should be retained on the CE-FMCI. Related to fluid dynamics, she clearly agreed that momentum was addressed in the course, since she indicated to "retain" item No. 6 (Figure 1B). However, the student was unsure about the applicability of item No. 25 (Figure 1D), stating that "This was really confusing, but we did learn forces in systems." Consequently, she did not identify that she could answer this question based on conservation of momentum.

\section{Discrepancies with Expert Judges}

The CE student differed from the judges on her evaluation of some compressible flow problems (Table 4). First, she failed to recognize that analysis of a gas in a manometer cannot be handled in the same manner as an incompressible liquid (No. 10). Second, she suggested that change in gas temperature and pressure caused by a variable area pipe was within the scope of a CE fluids course $($ No. 13, 31). While students may be able to answer this question using knowledge of the ideal gas law from physics, these topics are likely not discussed in depth in a CE fluids course.

Finally, there were some discrepancies related to boundary layer problems (Table 4). The student indicated that item No. 16 (Figure 2C), as well as the similar item No. 21, be removed from the future concept inventory. While judges debated about the applicability of these items, they eventually agreed to retain them on the CE-FMCI, since students could answer them based on knowledge of shear stress and shear strain. Clearly the relevance of these boundary layer items $($ No. 16, 21) is somewhat debatable. 
Table 4. Civil engineering student performance on the existing fluid mechanics concept inventory ${ }^{1}$.

\begin{tabular}{|c|c|c|c|c|c|c|c|}
\hline \multicolumn{2}{|r|}{ Item } & \multicolumn{2}{|c|}{$\begin{array}{c}\text { Percent Correct } \\
(\%)\end{array}$} & \multicolumn{2}{|c|}{ McNemar Test } & \multirow[t]{2}{*}{$\begin{array}{c}\text { Judges' } \\
\text { Consensus }\end{array}$} & \multirow[t]{2}{*}{$\begin{array}{r}\text { Student } \\
\text { Response }\end{array}$} \\
\hline No. & Targeted Concepts & Pre & Post & Statistic & $p$ & & \\
\hline 3 & Continuity; compressible & 48.6 & 51.4 & 0.000 & 1.000 & Retain & Retain \\
\hline 4 & Bernoulli; incompressible & 2.9 & 51.4 & 13.47 & $0.000 * * *$ & Retain & Retain \\
\hline 5 & Boundary conditions & 31.4 & 51.4 & 2.400 & 0.118 & Omit & Omit \\
\hline 6 & Momentum; incompressible & 20.0 & 45.7 & 4.267 & $0.035^{*}$ & Retain & Retain \\
\hline 7 & Pressure definition & 34.3 & 65.7 & 7.692 & $0.003 * *$ & Retain & Retain \\
\hline 8 & Boundary layers & 37.1 & 31.4 & 0.062 & 0.804 & Omit & Omit \\
\hline 9 & Pascal's Law & 34.3 & 77.1 & 11.53 & $0.000 * * *$ & Retain & Retain \\
\hline 10 & Manometry; compressible & 22.9 & 17.1 & 0.100 & 0.754 & Omit & Retain \\
\hline 11 & Bernoulli; incompressible & 62.9 & 37.1 & 3.048 & 0.078 & Retain & Retain \\
\hline 12 & Forces on submerged surface & 22.9 & 31.4 & 0.444 & 0.508 & Retain & Retain \\
\hline 13 & Ideal Gas Law & 0.0 & 11.4 & 2.250 & 0.125 & Omit & Retain \\
\hline 14 & Manometry; compressible & 20.0 & 14.3 & 0.167 & 0.688 & Retain & Retain \\
\hline 15 & Shear stress; compressible & 25.7 & 14.3 & 0.643 & 0.424 & Omit & Omit \\
\hline 16 & Boundary layers & 20.0 & 25.7 & 0.125 & 0.727 & Retain & Omit \\
\hline 17 & Bernoulli; incompressible & 5.7 & 51.4 & 12.50 & $0.000 * * *$ & Retain & Retain \\
\hline 18 & Manometry; compressible & 22.9 & 8.6 & 1.778 & 0.180 & Retain & Retain \\
\hline 19 & Drag force; compressible & 17.1 & 5.7 & 1.125 & 0.289 & Omit & Omit \\
\hline 20 & Boundary layer; compressible & 25.7 & 45.7 & 2.769 & 0.092 & Omit & Omit \\
\hline 21 & Boundary layer; incompressible & 14.3 & 40.0 & 4.923 & $0.022 *$ & Retain & Omit \\
\hline 22 & Continuity; incompressible & 17.1 & 25.7 & 0.308 & 0.581 & Retain & Retain \\
\hline 23 & Continuity/Bernoulli; incompressible & 2.9 & 34.3 & 7.692 & $0.003 * *$ & Retain & Retain \\
\hline 24 & Boundary layer; compressible & 20.0 & 25.7 & 0.083 & 0.774 & Omit & Omit \\
\hline 25 & Impulse-momentum; incompressible & 80.0 & 54.3 & 3.765 & $0.049 *$ & Retain & Unsure $^{2}$ \\
\hline 26 & Boundary layer; compressible & 34.3 & 22.9 & 0.643 & 0.424 & Omit & Omit \\
\hline 27 & Continuity/Bernoulli; incompressible & 5.7 & 28.6 & 4.900 & $0.021 *$ & Retain & Retain \\
\hline 28 & Drag force; compressible & 57.1 & 60.0 & 0.000 & 1.000 & Omit & Omit \\
\hline 29 & Drag force; compressible & 31.4 & 37.1 & 0.100 & 0.754 & Omit & Omit \\
\hline 30 & Pressure measurement; compressible & 5.7 & 11.4 & 0.167 & 0.688 & Retain & Retain $^{3}$ \\
\hline 31 & Continuity/Temperature variations; compressible & 5.7 & 8.6 & 0.000 & 1.000 & Omit & Retain \\
\hline 32 & Fluid properties (viscosity) & 94.3 & 88.6 & 0.250 & 0.625 & Retain & Retain \\
\hline
\end{tabular}

${ }^{1}$ Overall learning gain [(Post-Pre)/(30 Questions - Pre $\left.) * 100\right]$ calculated as $11 \%$.

${ }^{2}$ Student commented: "This was really confusing, but we did learn forces in systems."

${ }^{3}$ Student commented: “...weird question, the picture was really confusing..."

$* p \leq 0.05 ; * * p \leq 0.01 ; * * * p \leq 0.001$ 
(A)

4. Water flows through a pipe and enters a section where the cross sectional area is smaller. Viscosity, friction, and gravitational effects are negligible. Circle the letter of the correct statement about the change in pressure $\mathrm{p}$ and average velocity $\mathrm{V}$.

A $P_{2}$ is less than $P_{1}$ and $V_{2}$ is less than $V_{1}$

B $P_{2}$ is less than $P_{1}$ and $V_{2}$ is greater than $V_{1}$

C $P_{2}$ is greater than $P_{1}$ and $V_{2}$ is less than $V_{1}$

D $P_{2}$ is greater than $P_{1}$ and $V_{2}$ is greater than $V_{1}$

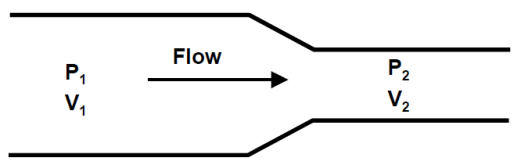

(C)

16. The plate of a large horizontal slider bearing moves at a steady speed over a layer of oil as shown in the figure below. There is no imposed pressure gradient in the direction of flow and the velocities in the oil are laminar and only due to the motion of the plate. Circle the letter that best represents the velocity profile (distribution of the velocity of the oil with distance) inside the fluid.

A. Profile A

B. Profile B

C. Profile C

D. Profile D

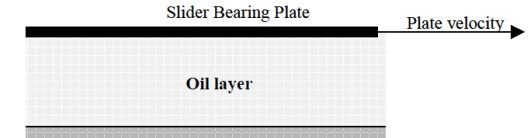

Stationary surface

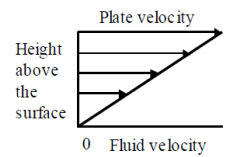

A

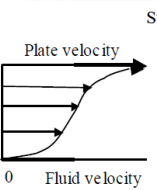

B

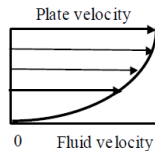

C

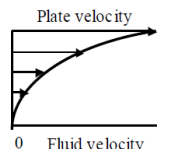

D
(D)

(B)

6. Two fluid jets are pointed at surfaces as shown in the figures below. The fluids are incompressible, and the effects of gravity can be neglected. The mass flow rate and the velocity of the jets are identical. The cross section area of the jets does not change significantly as the fluid flows. Circle the letter of the correct statement regarding the horizontal forces

A. $\mathrm{F}_{1}$ equals $2 \mathrm{~F}_{2}$

B. $F_{1}$ is greater than 0 and $F_{2}$ equals 0

C. $\mathrm{F}_{1}$ equals $\mathrm{F}_{2} / 2$

D. $F_{1}$ equals 0 and $F_{2}$ is greater than 0

E. $\mathrm{F}_{1}$ equals $\mathrm{F}_{2}$

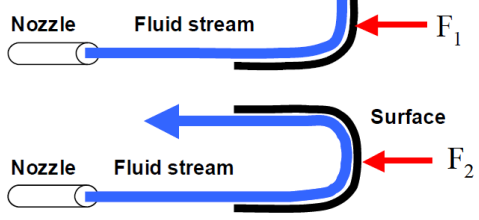

25. Two fluid jets are pointed at surfaces on the two carts as shown in the figures below. The fluids are incompressible, the effects of gravity can be neglected, the mass flow rate and the velocity of the jets are identical and the cross section area of the jets does not change as the fluid flows.. The carts move with a steady velocity in the directions shown below. The jet velocity is greater than the cart speed. Circle the letter of the correct statement.

A $F_{1}$ is greater than $F_{2}$

$B \quad F_{1}$ equals 0 and $F_{2}$ is greater than 0

C $F_{1}$ is less than $F_{2}$

D $F_{1}$ equals $F_{2}$ and both equal 0

E $F_{1}$ equals $F_{2}$

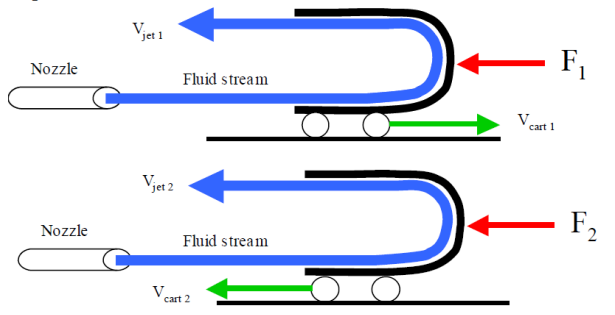

Figure 1. Sample existing FMCI items recommended to be retained on CE-FMCI (copied with permission from Martin et al. ${ }^{12}$ ). 
(A)

28. Circle the letter of the shape that has the highest form drag force (force due to pressure differences)
A. Shape A
B. Shape B
C. Shape C
D. Shape D
E. Shape E

(B)

24. Air flows over a flat plate as shown below. The flow is laminar and a boundary layer forms on the plate. Circle the letter of the statement that best represents the velocity profiles inside the boundary layer at the position 1 .

A. Velocity profile A

B. Velocity profile B

C. Velocity profile C

E. Velocity profile
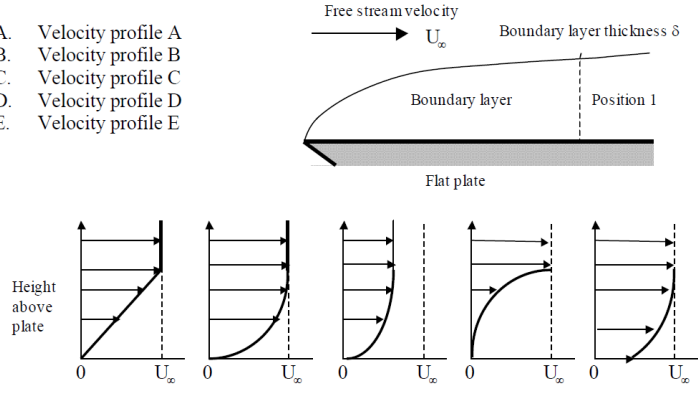

Velocity

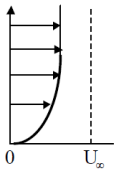

Velocity

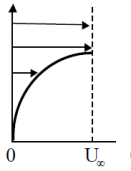

Velocity

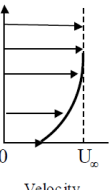

Shape A

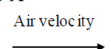

Air velocity

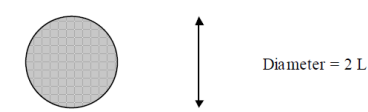

Shape B
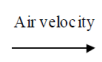

Shape C

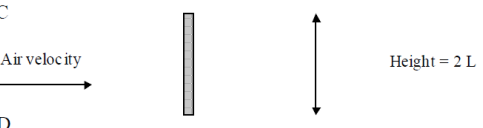

Shape D

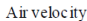

Shape E

$\stackrel{\text { Air velocity }}{\longrightarrow}$

(C)

31. Air flows through a well-insulated pipe and enters a section where the cross sectional area is larger. Viscosity, friction, and gravitational effects are negligible. Circle the letter of the correct statement regarding the temperatures and pressures of the air flow

A. The pressure $P_{2}$ equals $P_{1}$ and the temperature $T_{2}$ equals $T$

B. The pressure $P_{2}$ is greater than $P_{1}$ and the temperature $T_{2}$ is greater than $T_{1}$

C. The pressure $P_{2}$ is greater than $P_{1}$ and the temperature $T_{2}$ is less than $T_{1}$

D. The pressure $P_{2}$ is less than $P_{1}$ and the temperature $T_{2}$ is greater than $T_{1}$

E. The pressure $P_{2}$ is less than $P_{1}$ and the temperature $T_{2}$ is less than $T_{1}$

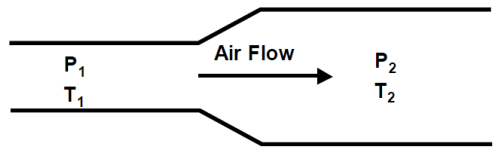

Figure 2. Sample existing FMCI items recommended to be omitted from CE-FMCI (copied with permission from Martin et al. ${ }^{12}$ ). 


\section{Discussion}

\section{Suggestions for Civil Engineering Fluid Mechanics Concept Inventory}

\section{Items to be Retained}

Despite the content and application differences between ME and CE fluid mechanics, there was consensus about several existing ME-FMCI items that should be retained on the CE-FMCI. For instance, judges and the student volunteer considered basic fluid properties, Bernoulli's/energy equation, conservation of mass, and conservation of momentum to be fundamental for civil engineers. Accordingly, students demonstrated significant learning gains for questions related to many of these topics (No. 4, 6, 7, 9, 17, 21, 23, 27) (Table 4). Although there was initially some disagreement among judges, consensus was eventually reached that some compressible flow (No. 14, 18, 30) and boundary layer (No. 16, 21) items should be represented on the CE-FMCI. The student evaluator included additional compressible flow problems on her "retain" list (No. $10,13,34)$, although successful completion of these problems would require skills clearly beyond the scope of a CE fluids course. She also suggested to "omit" boundary layer items No. 16 and 21. Overall agreement of the judges and the student was fairly good, considering that faculty and student insights into key concepts for inclusion on concept inventories is known to $\operatorname{differ}^{12}$.

Also notable is that improvements in student learning were not documented for several items deemed acceptable by study participants (e.g. No. 3, 11, 12) (Tables 3-4). Given that these questions clearly align with course objectives typical of a CE fluid mechanics course (Table 2), one possible explanation is that the course design was not conducive to student conceptual development. Literature supports that more traditional, instructor-centered pedagogies lead to only marginal improvements in conceptual knowledge ${ }^{35}$. Alternatively, students may have grown weary of answering unfamiliar, ME-related questions and did not exert their full effort. Other researchers have also highlighted the potential for student apathy toward concept inventory performance $^{38}$. Perhaps development of a CE-FMCI will aid in alleviating student fatigue during testing.

\section{Items to be Omitted}

Multiple items were recommended for omission from the CE-FMCI. All such questions involved some aspect of compressible flow, including boundary conditions (No. 20, 24, 26) and drag effects (No. 28, 29). Indeed, students did not demonstrate significant improvements in learning for any of the items that were omitted by the expert panel (Tables 3-4). However, student post-scores were impressively high for select items for which no class instruction was provided (No. 5, 20, 28) (Table 4). For instance, $60 \%$ of students were able to identify that rectangular objects experience higher drag forces than rounded objects (No. 28) (Figure 2A). Students could make this judgment based on the shape of airplanes, boat hulls, and other vehicles 
that are designed to minimize drag forces. Because student performance on these items would not be expected to improve after completion of a civil engineering fluids course, they should likely be omitted from the revised inventory.

\section{Items to be Created}

Important applications in CE should be reflected on the future CE-FMCI. For instance, experts agreed that concepts related to viscous flow, such as major/minor losses and use of pumps, should be included. Similarly, the group of ME faculty who originally developed the FMCI identified pipe networks as a "special case ${ }^{12 "}$ in fluid mechanics (pg. 24). Although the authors cite pipe networks as relevant to civil, chemical, and mechanical engineers, only one such problem is included on the ME-FMCI (No. 11). In addition, several judges in the current study also suggested that open channel flow concepts be included in a future revision. However, one judge presented an interesting point in the last round of the Delphi process that open channel flow may be more closely related with the content in a hydraulics course. Open channel flow was also listed by the founding ME-FMCI experts ${ }^{12}$, although it was noted to be only of interest to civil engineers.

\section{Implications for Practice}

Concept inventories can be used to assess student conceptual development for subsequent design and implication of classroom improvements. Overall, the learning gain (equation 1) for CE students in this study was $11 \%$ (Table 4 ), as compared to a range of $18 \%$ - 30\% for a group of ME students ${ }^{38}$. It is expected that ME students would perform better than CE students, given that the existing FMCI includes mechanical-engineering focused questions. Although these learning gains are relative, previous work cites learning gains of $30 \%$ are less are typical of predominately learning-centered classrooms ${ }^{30}$.

Several areas of improvement were identified based on CE student performance on the FMCI (Table 4). Students showed no statistical gains in learning related to static (No. 10, 14) and dynamic (No. 30) pressure measurement, as well as forces on static, submerged objects (No. 12) despite at least two weeks of instruction focused on such topics. Perhaps the in-class and homework problems were not sufficient for students to achieve a deep understanding of these topics. Alternatively, students may have forgotten concepts related to fluid statics, since this topic was covered early in the semester and not used extensively in subsequent discussions.

In addition, student knowledge of momentum may be limited. It is peculiar that significant improvements were documented for item No. 6 (Figure 1B) but not for item No. 25 (Figure 1D). In fact, even the high-performing student evaluator was unable to identify that item No. 25 is related to conservation of momentum. In contrast, previous researchers reported ME students at the University of Wisconsin to demonstrate some of the greatest gains for questions related to 
(ideal) conservation of momentum ${ }^{38}$. Perhaps there may be differences in student understanding of certain topics between $\mathrm{CE}$ and ME students.

Given the performance of students at the study institution, efforts are needed to improve student understanding of fluid statics and momentum. Perhaps the use of demonstrations, case studies, or other active strategies, as suggested by others ${ }^{35}$, would help students integrate these topics into their existing knowledge structures. In addition, re-introducing fluid statics at the end of the semester, perhaps as part of a Fundamentals of Engineering Exam review would remind students of topics addressed at the beginning of the semester.

\section{Implications for Research}

Since this is a study to complete only the preliminary steps of developing a CE-FMCI, there are numerous implications for future research. First, the Delphi process could be repeated by faculty at other institutions, and perhaps even practitioners, to capture input from a broader group on the applicability of some items that were initially contested in the current study related to compressible flow (e.g. No. 10,31) and boundary layer (No. 16, 21). Furthermore, drawing upon the expertise of others may allow for development of a more robust list of CE-specific topics, as well as constructive debate about the extent to which open channel flow should be represented on the CE-FMCI. Afterward, work is needed to create valid and reliable CE-specific items based on the guidelines outlined by previous developers of concept inventories. Once a CE-FMCI has been developed, the instrument can be used to quantify the impacts of educational interventions,

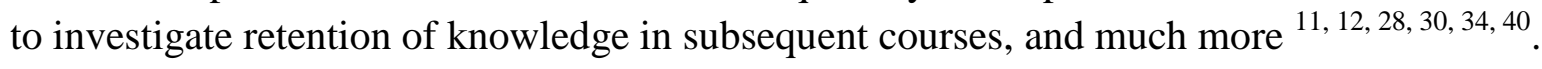

\section{Conclusions}

A study was conducted to complete preliminary steps necessary to modify the ME-FMCI for use in CE courses. A structured-communication process was used to facilitate a group of fluid mechanics content experts in reaching consensus about the applicability of existing ME-FMCI items for a future CE-FMCI. Civil engineering student input and performance on the ME-FMCI were used to validate expert consensus. The following conclusions were made based on the results.

1. According to expert and student consensus, existing ME-FMCI items related to basic fluid properties and definitions, conservation of mass, conservation of momentum, and Bernoulli's/energy equation should, without a doubt, be included on a CE-FMCI.

2. Although it was concluded that compressible flow and boundary effects should be included on a CE-FMCI to a limited extent, experts agreed that most of these problems should be omitted. 
3. Student performance on the ME-FMCI generally validated expert consensus, although any discrepancies are attributed to limitations of the course itself and/or student apathy during the testing process.

4. Future development of CE-FMCI items should include viscous flow in pipes, and perhaps open-channel flow.

Development of a CE-FMCI will provide engineering educators with a tool for quickly assessing student conceptual development in fluid mechanics courses. Consequently, instructors will be able to collect reliable data needed to make informed decisions about the design and improvement of their courses. Concerted efforts among educators from across courses in civil engineering curricula will likely contribute to the development of future engineers with complex, interrelated knowledge networks. Such deep conceptual understanding will facilitate the critical thinking and problem solving needed to design innovative strategies to society's ever-growing infrastructure challenges.

\section{References}

1. Davidson, C.I., et al., Viewpoint: Adding sustainability to the engineer's toolbox: A challenge for engineering educators. Environmental Science \& Technology, 2007. 41(14): p. 4847-4849.

2. Montfort, D., S. Brown, and D. Pollock, An investigation of students' conceptual understanding in related sophomore to graduate-level engineering and mechanics courses. Journal of Engineering Education, 2009. 98(2): p. 111-129.

3. Streveler, R.A., et al., Learning conceptual knowledge in the engineering sciences: Overview and future research directions. Journal of Engineering Education, 2008. 97(3): p. 279-294.

4. Sheppard, S., et al., What is engineering practice? International Journal of Engineering Education, 2007. 22(3): p. 429.

5. Freeman, S., et al., Active learning increases student performance in science, engineering, and mathematics. Proceedings of the National Academy of Sciences, 2014. 111(23): p. 8410-8415.

6. Prince, M.J. and R.M. Felder, Inductive teaching and learning methods: Definitions, comparisons, and research bases. Journal of Engineering Education, 2006. 95(2): p. 123-138.

7. Bhattacharya, K. and S. Han, Piaget and cognitive development, in Emerging Perspectives on Learning, Teaching, and Technology, M. Orey, Editor. 2001, University of Georgia: Athens, GA.

8. Eggen, P. and D. Kauchak, Educational Psycology: Windows on Classrooms. 8th ed. 2010, Upper Saddle River, NJ: Pearson.

9. Cobb, P. and E. Yacke, Constructivist, emergent, and socialcultural perspectives in the context of developmental research. Educational Psychologist, 1996. 31(3/4): p. 175-190.

10. Chi, M.T., Three types of conceptual change: Belief revision, mental model transformation, and categorical shift. International handbook of research on conceptual change, 2008: p. 61-82.

11. Streveler, R.A., et al., Rigorous methodology for concept inventory development: Using the'assessment triangle'to develop and test the thermal and transport science concept inventory (TTCI). International Journal of Engineering Education, 2011. 27(5): p. 968.

12. Martin, J., J. Mitchell, and T. Newell. Development of a concept inventory for fluid mechanics. in Frontiers in Education, 2003. FIE 2003 33rd Annual. 2003. IEEE.

13. Evans, D., et al. Progress on concept inventory assessment tools. in Frontiers in Education, 2003. FIE 2003 33rd Annual. 2003. IEEE.

14. Hsu, C.-C. and B.A. Sandford, The Delphi technique: making sense of consensus. Practical assessment, research \& evaluation, 2007. 12(10): p. 1-8. 
15. Okoli, C. and S.D. Pawlowski, The Delphi method as a research tool: an example, design considerations and applications. Information \& Management, 2004. 42(1): p. 15-29.

16. Dalkey, N. and O. Helmer, An experimental application of the Delphi method to the use of experts. Management Science, 1963. 9(3): p. 458-467.

17. Baroody, A.J., Y. Feil, and A.R. Johnson, An alternative reconceptualization of procedural and conceptual knowledge. Journal for Research in Mathematics Education, 2007. 38: p. 115-131.

18. Rittle-Johnson, B., Promoting Transfer: Effects of Self-Explanation and Direct Instruction. Child Development, 2006. 77(1): p. 1-15.

19. Star, J.R., Reconceptualizing procedural knowledge. Journal for Research in Mathematics Education, 2005. 36: p. 404-411.

20. Bransford, J., et al., How people learn : Brain, mind, experience, and school. 2000, Washington, D.C.: National Academy Press.

21. Alexander, P.A. and P.K. Murphy, Nurturing the seeds of transfer: A domain-specific perspective. International Journal of Educational Research, 1999. 31(7): p. 561-576.

22. Chi, M.T. and R.D. Roscoe, The processes and challenges of conceptual change, in Reconsidering conceptual change: Issues in theory and practice. 2002, Springer. p. 3-27.

23. Rittle-Johnson, B., R.S. Siegler, and M.W. Alibali, Developing conceptual understanding and procedural skill in mathematics: An iterative process. Journal of educational psychology, 2001. 93(2): p. 346.

24. Piaget, J., M. Cook, and W. Norton, The origins of intelligence in children. Vol. 8. 1952: International Universities Press New York.

25. Limón, M. and L. Mason, Reconsidering conceptual change: Issues in theory and practice. 2002: Springer.

26. Chi, M.T., J.D. Slotta, and N. De Leeuw, From things to processes: A theory of conceptual change for learning science concepts. Learning and instruction, 1994. 4(1): p. 27-43.

27. Carey, S., Knowledge acquisition: Enrichment or conceptual change. Concepts: core readings, 1999: p. 459-487.

28. Prince, M., M. Vigeant, and K. Nottis, Development of the heat and energy concept inventory: Preliminary results on the prevalence and persistence of engineering students' misconceptions. Journal of Engineering Education, 2012. 101(3): p. 412-438.

29. Halloun, I.A. and D. Hestenes, The initial knowledge state of college physics students. American journal of Physics, 1985. 53(11): p. 1043-1055.

30. Gray, G.L., et al. Toward a nationwide dynamics concept inventory assessment test. in American Society for Engineering Education Annual Conference \& Exposition. 2003.

31. Concept Inventories. 200131 January 2015]; Available from: http://www.foundationcoalition.org/home/keycomponents/concept/.

32. Rossouw, A., M. Hacker, and M.J. de Vries, Concepts and contexts in engineering and technology education: An international and interdisciplinary Delphi study. International Journal of Technology and Design Education, 2011. 21(4): p. 409-424.

33. Streveler, R.A., et al. Using a Delphi study to identify the most difficult concepts for students to master in thermal and transport science. in Proceedings of the Annual Conference of the American Society for Engineering Education. 2003.

34. Olds, B.M., et al. Preliminary results from the development of a concept inventory in thermal and transport science. in CD) Proceedings, 2004 American Society for Engineering Education Conference. 2004.

35. Hake, R.R., Interactive-engagement versus traditional methods: A six-thousand-student survey of mechanics test data for introductory physics courses. American journal of Physics, 1998. 66(1): p. 64-74.

36. McDermott, L.C., Physics by Inquiry, Volume 1. Physics by Inquiry, Volume 1, by Lillian C. McDermott, Physics Education Group, Univ. of Washington, pp. 400. ISBN 0-471-14440-1. Wiley-VCH, August 1995., 1995. 1.

37. Atilola, O., et al. Automatic Identification of Student Misconceptions and Errors for Truss Analysis. in American Society for Engineering Education. 2012. American Society for Engineering Education.

38. Mitchell, J.W. and J.K. Martin, Use of thermodynamics, fluid mechanics and heat transfer concept inventories to assess student learning in core courses. University of Wisconsin: Madison, WI.

39. Sheskin, D.J., Handbook of parametric and nonparametric statistical procedures. 2003: crc Press.

40. Jacobi, A., et al. A concept inventory for heat transfer. in Frontiers in Education, 2003. FIE 2003 33rd Annual. 2003. IEEE. 